\title{
COMMUNICATION
}

\section{Fabrication of Ultra-thin Polyrotaxane-based Films via Solid-state Continuous Assembly of Polymers}

Cite this: DOI: $10.1039 / \times 0 \times x 00000 x$

Received 00th January 2012,

Accepted 00th January 2012

DOI: $10.1039 / \times 0 \times x 00000 x$

www.rsc.org/

\author{
Shereen Tan, ${ }^{a}$ Eunhyung Nam, ${ }^{a}$ Jiwei Cui, ${ }^{a}$ Chenglong Xu, ${ }^{a}$ Qiang Fu, ${ }^{a}$ Jing M. Ren, ${ }^{a}$ \\ Edgar H. H. Wong, ${ }^{a}$ Katharina Ladewig, ${ }^{a}$ Frank Caruso, ${ }^{a}$ Anton Blencowe,,${ }^{b, *}$ Greg \\ G. Qiao ${ }^{a, *}$
}

Surface-confined ultra-thin polyrotaxane (PRX)-based films with tunable composition, surface topology and swelling characteristics were prepared by solid-state continuous assembly of polymers (ssCAP). The PRX-based films supported cell attachment, and their degradation in biological media could be tuned. This study provides a versatile nano-coating technology with potential applications in biomedicine, including tissue engineering and medical devices.

The self-assembly of complex multi-molecular systems has received expanding interest in both organic and materials chemistry. ${ }^{1}$ This is attributed to the precise nature of the interactions, which leads to functional constructs that are able to rapidly respond to stimuli. ${ }^{1 \mathrm{~b}}$ Inclusion chemistry, wherein a 'host' reversibly binds to a 'guest' is a self-assembly process capable of forming highly ordered reversible constructs. ${ }^{2}$ Of particular note are cyclodextrin (CD)based PRXs first discovered by Harada and co-workers. ${ }^{3}$ CD-based PRXs are synthesised by threading numerous CD moieties onto a polymeric backbone (e.g., PEG), and subsequently end-capping the polymer with bulky end groups to prevent dethreading. ${ }^{2 b, 3 a, b}$ This allows the supramolecular construct to gain both rotational and translational freedom (via the CDs) without disassociation. ${ }^{4}$ Since the inception of these constructs, a variety of sophisticated multicomponent supramolecular assemblies ${ }^{2 \mathrm{~b}, 5}$ and advanced functional materials ${ }^{6}$ have been fabricated using PRXs and their derivatives as building blocks. PRXs have been used for a wide range of applications, including $\operatorname{drug}^{7}$ and gene ${ }^{8}$ delivery systems, scaffolds for tissue engineering, ' highly elastic yet tough 'sliding-ring' materials, ${ }^{10}$ and insulated molecular wires. ${ }^{11}$ Although much work has focused on the development and characterisation of macroscale CD-based PRX constructs, the study of nanoscale CD-based PRX functional films remains largely unexplored, with only several reports in the literature. ${ }^{7 \mathrm{a}, 12 \mathrm{a}, \mathrm{b}}$ Furthermore, the incorporation of stimuli-responsive polymers may enable triggered release of therapeutics. ${ }^{12 c, d}$ Recently, we reported the build-up of degradable PRX multilayer films using the layer-by-layer (LbL) technique based upon electrostatic interactions. ${ }^{12 a}$ The ability to initiate film degradation under biological conditions and the potential to conjugate various drugs and biomolecules onto the CD films highlight their potential in the biomedical field. However, the ability to tailor the composition and physical properties of these films is complicated by the necessity to have complementary polyelectrolytes. Therefore, novel strategies that provide facile access to PRX-based nanoscale films with tunable properties (i.e., surface morphology, swellability and elasticity) are highly desirable, particularly for biointerfaces whereby cellular interactions and behaviour are dependent on the composition, topography and mechanical properties of the film. ${ }^{13}$

Herein, we report the synthesis of ultra-thin $(<100 \mathrm{~nm})$ PRXbased cross-linked films using a PRX macrocross-linker in combination with the recently developed solid-state continuous assembly of polymers (ssCAP) technique (Scheme 1$).{ }^{14}$ Through the simple addition of selected additives during film fabrication, the composition, surface topography, swelling characteristics and degradation rate of the nanoscale PRX films could be controlled. Although predominately composed of low fouling PEG, ${ }^{15}$ the PRXbased films were able to facilitate cell attachment and proliferation, which was attributed to the heterogeneity of the PRXs. The ability of the ssCAP technique to produce multi-component PRX-based films with engineered properties makes this approach applicable to the fabrication of functional nanobiotechnology coatings.
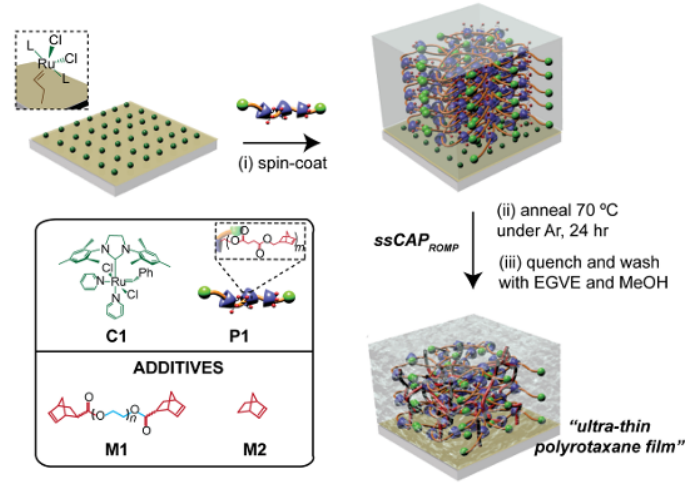

Scheme 1. Synthesis of ultra-thin PRX-based films via ssCAP ROMP. The physical properties of the resulting films were tailored by the addition of selected additives, including the norbornene endfunctionalized PEG M1 or the monomer norbornene M2. 
The ultra-thin PRX-based films were prepared via the ssCAP approach (Scheme 1) as attempts to prepare analogous films from solution state CAP approachs ${ }^{16}$ were unsuccessful. Ring-opening metathesis polymerization (ROMP) was used to mediate the ssCAP $_{\text {ROMP }}$ process to afford surface-confined PRX-based films, whereby the PRX P1 acts as a macrocross-linker. Film formation was performed on silicon wafers that were pre-functionalised with an initiating layer of the ruthenium $(\mathrm{Ru})$ catalyst $\mathbf{C 1}$ (refer to Supporting Information (SI) for details). The PRX macrocross-linker $\mathbf{P 1}$, with and without additives (PEG cross-linker M1 or norbornene M2), were deposited onto the substrate via spin-coating, which resulted in a $60 \mathrm{~nm}$ film (SI, Fig. S1). Subsequently, the films were annealed under an argon atmosphere at $70{ }^{\circ} \mathrm{C}$ (i.e., above the melting temperature $\left(T_{\mathrm{m}}\right)$ of $\mathbf{P 1}$ (SI, Fig. S2)) for $24 \mathrm{~h}$ (Scheme 1). The resultant cross-linked films were then washed in methanol containing di(ethylene glycol) vinyl ether (EGVE) to detach the Ru catalyst from the film and to remove any uncross-linked material prior to analysis. Multilayer films were prepared using a previously described re-initiation method. ${ }^{14,16}$

The PRX-based macrocross-linker P1 was synthesised in two steps by slight modification of methods reported in the literature. ${ }^{17}$ Briefly, $\alpha, \omega$-dialkyne PEG $_{10 \mathrm{~K}}$ was threaded with $\alpha$-CDs to yield a polypseduorotaxane as a precipitate. The product was lyophilised prior to end-capping via copper catalyzed azide-alkyne cycloaddition (CuAAC) with an azido-anthracene derivative. This resulted in PRXs bearing anthracene end-caps at the PEG chain ends. From ${ }^{1} \mathrm{H}$ NMR spectroscopic analysis, the inclusion ratio and the end-capping efficiency of the PRX were determined to be $c a$. 3 CDs per PEG chain and 80\%, respectively (SI, Fig. S3a). Polymerizable pendent norbornene groups were then conjugated onto the PRX by partial esterification of the primary hydroxyl groups of the threaded CDs with a norbornene acid derivative to afford PRX P1 (Scheme 1). ${ }^{1} \mathrm{H}$ NMR spectroscopic analysis of P1 (SI, Fig. S3b) revealed the presence of $c a$. $3 \mathrm{CD}$ moieties per PRX and an average of 3 polymerizable norbornene moieties per CD (i.e., $5 \mathrm{~mol} \%$ of norbornene relative to CD primary hydroxyl groups.

Thickness analysis of the PRX-based films was conducted via atomic force microscopy (AFM). Fig. 1A illustrates the growth profile of a range of nanoscale PRX-based films consisting of various weight percentages (wt\%) of $\mathbf{P 1}$ with and without additives (M1 or M2), with respect to the polymeric layer (L) (where L1 denotes the first ssCAP reaction from the initial Ru-modified $\mathrm{Si}$ substrate). For the L1 film prepared from the PRX macrocross-linker P1 alone, the initial film thickness was determined to be $c a .10 \mathrm{~nm}$. Upon re-initiation (L2-L4), the thickness of PRX P1 based films increased by ca. $6 \mathrm{~nm}$ per layer to yield an ca. $25 \mathrm{~nm}$ film after four layers (L4) (Fig. 1A). This is in contrast to control experiments (i.e., $\mathbf{P 1}$ annealed in the absence of catalyst $\mathbf{C 1}$ ) that provided a thickness of $c a .2 .5 \mathrm{~nm}$, which was attributed to the physical adsorption of polymers (SI, Fig. S4). The surface roughness (Fig. 1B) of the P1 film was found to increase significantly with each additional layer (L1: $1.5 \mathrm{~nm}$ to L4: $25.7 \mathrm{~nm}$ ), which correlated to the presence of relatively large particles with diameters ranging from $c a$. 1-1.4 $\mu \mathrm{m}$ was observed on the surface topography of the air-dried films (SI, Fig. S5, $\mathbf{P 1}$ dry-state). The presence of such particles was attributed to the aggregation of the PRXs as previously reported. ${ }^{12 a}$ To assess whether the bulky threaded CDs of P1 influence film growth, ssCAP $_{\mathrm{ROMP}}$ films were also prepared from a linear PEG macrocrosslinker $\mathbf{P} 2$ with pendent norbornene groups (5 mol \% with respect to total amount of hydroxyl groups) (Fig. 2A). Both $\mathbf{P 1}$ and the linear PEG macrocross-linker $\mathbf{P} 2$ have approximately the same amount of hydroxyl and norbornene groups as evaluated by ${ }^{1} \mathrm{H}$ NMR spectroscopy (SI, Fig. S3b and S6, respectively). Whereas P1 provided a film thickness of $c a .10 \mathrm{~nm}$ after one layer (Fig. 1A), the film derived from $\mathbf{P} 2$ was significantly thicker (L1; ca. 80 nm, Fig. S7). The significant difference in film growth was attributed to the steric hindrance of the bulky CDs decreasing accessibility to the norbornenes (i.e., polymerizable moieties) during the ssCAP process. Based upon these observations, it was rationalised that the addition of relatively small, mobile and polymerisable additives during the CAP process with $\mathbf{P 1}$ would improve film growth, as well as providing an additional handle to control the films properties. Therefore, the addition of the PEG-based cross-linker $\mathbf{M 1}$ as an additive was studied. M1 acts as a brush-like spacer, increasing the intermolecular distance between adjacent cross-linkable PRXs whilst also creating additional cross-linking points. Whereas the addition of 20 wt\% of $\mathbf{M 1}$ had negligible effect on the P1 film thickness over multiple layers relative to $\mathbf{P 1}$ alone (Fig. 1), the addition of $50 \mathrm{wt} \%$ of $\mathbf{M 1}$ resulted in an increase of $15 \mathrm{~nm}$ per layer after the first layer (L1; $8 \mathrm{~nm}$ ) was deposited (Fig. 1A). Overall, the addition of $50 \mathrm{wt} \%$ M1 assisted film growth, resulting in a 2.5 fold increase in film thickness per layer as compared to P1 alone. Furthermore, the surface topography of the films prepared with M1 (20 and $50 \mathrm{wt} \%$ ) appeared smoother (c.f., P1 films), which was attributed to the flexible nature of the PEG chains (SI, Fig. S5, P1+M1 (20 and $50 \mathrm{wt}$ \%) dry-state). The monomer, norbornene M2, was also selected as an additive for film formation as it may act as a spacer, increasing the intermolecular distance between PRXs. Although the addition of $50 \mathrm{wt} \% \mathbf{M} 2$ did not seem to improve film growth compared to P1 alone (Fig. 1A), a significant decrease in film roughness (Fig. 1B) was noted (SI, Fig. S5, P1+M2 (50 wt\%) dry state). This result is attributed to the ability of free norbornene to connect neighbouring P1 molecules and occupy voids, thereby resulting in more homogenous films similar to poly(norbornene) brushes synthesised via grafting-from techniques. ${ }^{18}$
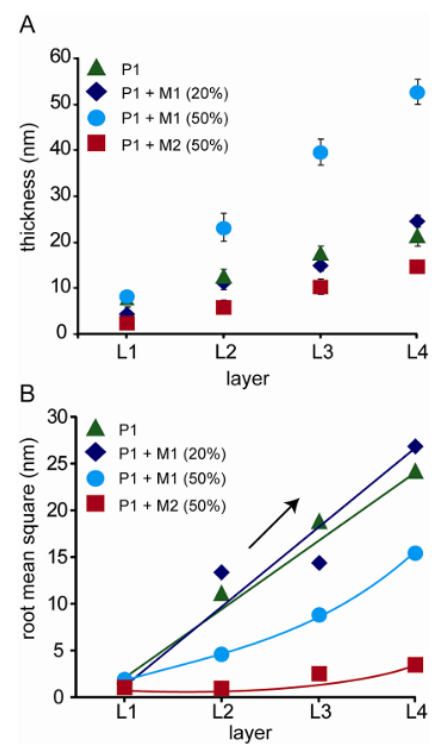

Figure 1. Air-dried PRX-based nanoscale films made via ssCAP $_{\text {ROMP }}$ in the presence of $\mathbf{P 1}$ and different amounts (wt\%) of additives (M1 or M2). (A) 'Air-dried' film thickness with polymeric layer (L) on planar silica (Si) substrates, as measured in-air by AFM. (B) Film surface roughness (root mean square) with increasing polymeric layer (L), as measured by dry-state AFM. Note, the plotted film thickness values are based upon the measured film thicknesses minus control experiments (that measured physical adsorption), and are the average of 15 independent measurements.

The PRX P1-based nanoscale films obtained after three reinitiations (i.e., L4) were subjected to swelling experiments, whereby 
the films were swollen in water for 2 days. The degree of swelling was measured in terms of the equilibrium swelling ratio (ESR \%) (SI, Equation 1) using thickness measurements obtained from dry and wet-state AFM analysis (SI, Fig. S8). The addition of additives (M1 or M2) is expected to increase the intermolecular distance between the CD cross-link points, thereby decreasing cross-linking density and allowing for greater swelling of the films in comparison to films fabricated solely from $\mathbf{P 1}$.

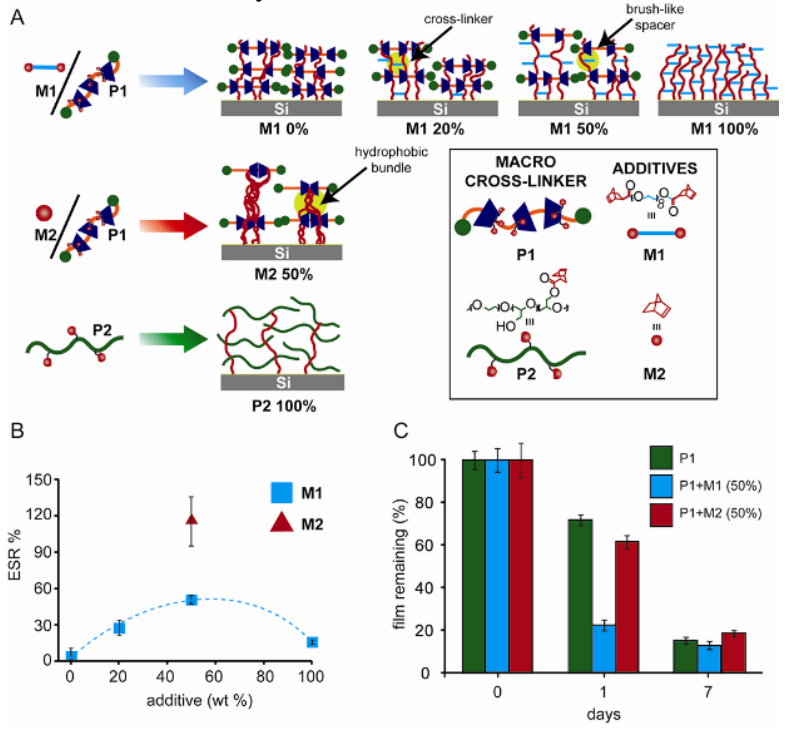

Figure 2. (A) Illustration of the PRX-based nanoscale film (L4) morphology in its fully swollen state when additives (M1 or M2) are incorporated at various weight percentages (wt\%). (B) Equilibrium swelling ratio (ESR\%) of the fully swollen PRX-based nanoscale films measured by AFM. (C) Degradation studies of various films submerged in a mixture of $10 \%$ blood serum and $90 \%$ PBS over 7 days. Percentage degradation was taken as the reduction in film thickness, as measured by wet-state AFM over time.

Fig. 2A is a theoretical illustration of the change in film morphology of a series of swollen PRX-based nanoscale films with different amounts of additives (M1 or M2). It was observed that films prepared with just the PRX macrocross-linker P1 did not swell significantly (ESR\% ca. 2.0\%, Fig. 2B) as the distance between the CD cross-link points would be very short. Furthermore, as the CDs are significantly larger than the norbornene groups, it is also possible that the ROMP process was sterically hindered. However, when the PEG cross-linker M1 is introduced, the distance between the CD cross-link points is increased as the PEG acts as a brush-like spacer decreasing the cross-link density of the film (Fig. 2A). Therefore, the incorporation of $\mathbf{M 1}$ (20 and 50 wt\%) into P1 films results in films with ESR\% values of $c a .30$ and $50 \%$, respectively (Fig. 2B). The increase in ESR\% is attributed to a decrease in cross-linking density between adjacent PRXs molecules. For comparison, films prepared solely from M1 were also analysed, and displayed lower swellability (ESR\% of $c a .16 \%$, Fig. 2B) than films prepared from both $\mathbf{P 1}$ and M1, which is attributed to a higher cross-linking density. Although PEG is generally hydrophilic in nature, the high cross-linking density and low swellability of pure M1 (i.e., PEG $_{400 \mathrm{Da}}$ ) films most likely results from the low molecular weight of M1 and high norbornene to ethylene glycol repeat unit ratio (2:9). When the molecular weight of PEG is significantly increased (e.g., with the use of $\mathrm{PEG}_{20 \mathrm{kDa}} \mathbf{P}$ 2, Fig. 2A) leading to a decrease in the norbornene to ethylene glycol repeat unit ratio, the resulting films fabricated via the same ssCAP process with only one layer are able to swell significantly more (i.e., ESR \% ca. 167 \%, Fig. S9). The significant increase in swellability compared to pure M1 films is attributed to the lower cross-linking density (Fig. 2A). It is expected that films fabricated with $\mathbf{M 1}$ and $\mathbf{P 1}$ would possess an increased intermolecular spacing between PRXs resulting from the presence of PEG-based brush-like spacers (Fig. 2A). However, owing to the difunctional nature of M1, additional covalent cross-linking sites are also introduced, which contributes to the film cross-linking density, thereby limiting the swellability of the films. To avoid this scenario, the monomer, norbornene M2, was added as an additive at $50 \mathrm{wt} \%$. As M2 is a monomer, it was expected that the norbornene groups would act solely as a spacer increasing the intermolecular distance between PRXs (Fig. 2A). It was observed that films prepared with $50 \%$ M2 were able to swell significantly more than any other PRXbased films fabricated in the series (ESR \% $116 \%$, Refer to Fig. $2 \mathrm{~B}$, red icon). The significant increase in ESR is suggestively attributed to the hydrophobic polynorbornene chains bundling together in a collapsed configuration and creating hydrated pockets throughout the polymeric network. In addition, it is expected that the threaded CDs moieties would slide along the PEG backbone and aggregate. The aggregation of the CDs thus exposes the hydrophilic PEG backbone. These two contributing factors are expected to allow for higher swellability than the other PRX-based films (Fig. 2A and B).

The degradability of the PRX-based nanoscale films was also studied by immersing the films in a mixture of $10 \%$ blood plasma and $90 \%$ PBS. As serum contains a variety of enzymes, including those capable of catalysing hydrolyses of ester bonds, it was hypothesised that the degradation of the PRX films could be tailored by controlling the type and amount of additive (M1 or M2) incorporated. Degradation of the films was measured by thickness analysis at various time points using wet-state AFM (Fig. S10). It was observed that PRX films fabricated with additive M1 (50 wt\%) displayed the highest percentage of degradation (Fig. 2C) after $1 \mathrm{~d}$, which was attributed to the higher content of ester bonds present in the M1 component. Near complete degradation (> 80\%) of all the films was observed after 7 days. The ability to tune the film degradation rate further demonstrates the potential of these films as nano-coatings for various bio-related applications. Thus, by varying the type and amount of additive used, the physical properties of the PRX-based films can be tailored.

Functional materials composed of $\alpha$-CD/PEG PRXs have shown potential to control and tune the extent of cell adhesion and proliferation. ${ }^{9}$ For example, the incorporation of PRXs into PEGbased hydrogels has led to successful cell attachment and proliferation. ${ }^{9}$ This phenomenon is believed to result from the surface heterogeneity generated by the PRXs, rather than the chemical composition of the CD groups. ${ }^{9}$ The ability to control heterogeneity by simply varying the amount of PRXs, or the amount of threaded CDs, makes PRX-based materials and interfaces potential candidates for tissue engineering and biomedical coating applications. Therefore, the cell adhesion characteristics of the nanoscale PRX-based film composed purely of P1 was investigated using mouse embryonic fibroblast (NIH/3T3) cells according to our previously reported assay. ${ }^{19}$ Non-modified and PEG-coated (i.e., P2coated) glass slides were used as positive and negative controls, respectively, and cell attachment was quantified by acquiring 10 images from random locations on each substrate using fluorescent microscopy (Fig. 3 and SI, Fig. S11).

Unlike the $\mathbf{P} 2$ PEG films, P1-based films were able to support cell adhesion and proliferation, as observed via fluorescence microscopy (Fig. 3). The ability of inherently rough $\mathbf{P 1}$ based films to support cell attachment and proliferation was attributed to the heterogeneity of the PRXs, despite the films being predominately composed of PEG (i.e., main-chain of the PRX is PEG (ca. 75 wt. \%)), which is 
low fouling (Fig. 3). The relatively high content of PEG in the P1 films is also believed to contribute to the $50 \%$ reduction in cell adhesion in comparison to the glass slides (Fig. 3). Interestingly, when cell adhesion studies were conducted using HeLa cell lines, no cell attachment was observed for both PRX P1 and PEG based films. These promising preliminary results indicate that the ultra-thin PRXbased films could potentially be employed as nano-coatings in various biomedical applications. In addition, the remaining hydroxyl groups on the CD cross-links within the PRX networks could also be used to support the conjugation of various receptors and biofactors that facilitate specific cell interactions and behaviour. The dynamic nature of CDs conjugated with specific receptors further increases the potential of these materials as increased ligand affinity is often seen owing to their dynamic and multivalent nature. ${ }^{20}$

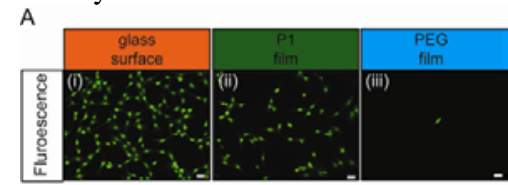

B

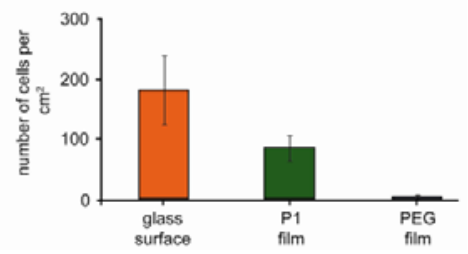

Figure 3. (A) Cell (NIH/3T3) attachment on glass, P1 ssCAP $\mathrm{ROMP}_{\mathrm{P}}$ film (L4) and $\mathbf{P} 2$ ssCAP $_{\text {ROMP }}$ film (L1). (B) Number of cells per surface area attached on the respective substrates. Scale bars are 100 $\mu \mathrm{m}$.

In conclusion, ultra-thin PRX-based networks were fabricated using $\mathrm{CD} / \mathrm{PEG} \mathrm{PRXs}$ as the building block via the recently developed ssCAP $_{\text {ROMP }}$ technique. Using this approach, the composition and resulting physical properties of the PRX-based films could be tuned by the addition of various additives during the fabrication process. In addition, the rate of film degradation in the presence of blood serum was also demonstrated and could be controlled by the choice of additive. Preliminary cell attachment studies using NIH/3T3 cell lines indicated that the nanoscale PRXbased films were also able to support cell adhesion, highlighting their potential application in the field of nanobiotechnology. These promising results highlight the ability of the ssCAP approach to construct multi-compositional PRX films with tunable properties on the nanoscale, providing new avenues for PRX-based technologies to be applied as nano-coatings.

The authors acknowledge the Australian Research Council under the Future Fellowship Scheme (FT110100411, G.G.Q.) and Discovery Project (DP1094147 and DP130101846, F.C., G.G.Q). K. L., Q.F. and J. C. are the recipients of the Australian Research Council Super Science Fellowship (FS110200025).

\section{Notes and references}

${ }^{a}$ Department of Chemical and Biomolecular Engineering, The University of Melbourne, Parkville, Victoria 3010, Australia

${ }^{b}$ Mawson Institute, Division of ITEE, The University of South Australia, Mawson Lakes, SA 5095, Australia

$\dagger$ Electronic Supplementary Information (ESI) available: [details of any supplementary information available should be included here]. See DOI: 10.1039/c000000x/
1. (a) G. Chen and M. Jiang, Chemical Society Reviews, 2011, 40, 22542266; (b) D. A. Uhlenheuer, K. Petkau and L. Brunsveld, Chemical Society Reviews, 2010, 39, 2817-2826; (c) O. Ikkala and G. ten Brinke, Science, 2002, 295, 2407-2409.

2. (a) F. van de Manakker, T. Vermonden, C. F. van Nostrum and W. E. Hennink, Biomacromolecules, 2009, 10, 3157-3175; (b) A. Harada, Y. Takashima and H. Yamaguchi, Chemical Society Reviews, 2009, 38, 875-882; (c) C. O. Mellet, J. M. G. Fernandez and J. M. Benito, Chemical Society Reviews, 2011, 40, 1586-1608; (d) S. Tan, K. Ladewig, Q. Fu, A. Blencowe and G. G. Qiao, Macromolecular Rapid Communications, 2014, 35, 1166-1184.

3. (a) A. Harada, J. Li and M. Kamachi, Nature, 1992, 356, 325-327; (b) H. Shigekawa, K. Miyake, J. Sumaoka, A. Harada and M. Komiyama, Journal of the American Chemical Society, 2000, 122, 5411-5412; (c) A. Harada, J. Li, T. Nakamitsu and M. Kamachi, The Journal of Organic Chemistry, 1993, 58, 7524-7528.

4. A. Harada, Accounts of Chemical Research, 2001, 34, 456-464.

5. (a) Q. Fu, J. M. Ren, S. Tan, J. Xu and G. G. Qiao, Macromolecular Rapid Communications, 2012, 33, 2109-2114; (b) S. Tan, E. H. H. Wong, Q. Fu, J. M. Ren, A. Sulistio, K. Ladewig, A. Blencowe and G. G. Qiao, Australian Journal of Chemistry, 2014, 67, 173-178.

6. J. Araki and K. Ito, Soft Matter, 2007, 3, 1456-1473.

7. (a) H. H. Dam and F. Caruso, ACS Nano, 2012, 6, 4686-4693; (b) S. Yu, Y. Zhang, X. Wang, X. Zhen, Z. Zhang, W. Wu and X. Jiang, Angewandte Chemie International Edition, 2013, 52, 7272-7277; (c) J. Li, F. Zhao and J. Li, Appl Microbiol Biotechnol, 2011, 90, 427-443.

8. T. Ooya, H. S. Choi, A. Yamashita, N. Yui, Y. Sugaya, A. Kano, A. Maruyama, H. Akita, R. Ito, K. Kogure and H. Harashima, Journal of the American Chemical Society, 2006, 128, 3852-3853.

9. (a) J. Watanabe, T. Ooya, K.-H. Nitta, K. D. Park, Y. H. Kim and N. Yui, Biomaterials, 2002, 23, 4041-4048; (b) W. K. Lee, T. Ichi, T. Ooya, T. Yamamoto, M. Katoh and N. Yui, Journal of Biomedical Materials Research Part A, 2003, 67A, 1087-1092.

10. (a) Y. Okumura and K. Ito, Advanced Materials, 2001, 13, 485-487; (b) J. Araki, T. Kataoka and K. Ito, Soft Matter, 2008, 4, 245-249; (c) K. Ito, Current Opinion in Solid State and Materials Science, 2010, 14, 28-34; (d) S. Tan, A. Blencowe, K. Ladewig and G. G. Qiao, Soft Matter, 2013, 9, 5239-5250.

11. F. Cacialli, J. S. Wilson, J. J. Michels, C. Daniel, C. Silva, R. H. Friend, N. Severin, P. Samori, J. P. Rabe, M. J. O'Connell, P. N. Taylor and H. L. Anderson, Nat Mater, 2002, 1, 160-164.

12. (a) H. H. Dam and F. Caruso, Advanced Materials, 2011, 23, 3026-3029; (b) G. Rydzek, T. Garnier, P. Schaaf, J.-C. Voegel, B. Senger, B. Frisch, Y. Haikel, C. Petit, G. Schlatter, L. Jierry and F. Boulmedais, Langmuir, 2013, 29, 10776-10784. (c) X. Chen, W. Wu, Z. Guo, J. Xin, J. Li, Biomaterials, 2011, 32, 1759-1766. (d) M. Nakahata, Y. Takashima, H. Yamaguchi. and A. Harada, Nat. Commun., 2011, 2, 511.

13. S.Ouasti, R. Donn, o, F. Cellesi, M. J. Sherratt, G. Terenghi and N. Tirell, Biomaterials, 2011, 32,6456-6470.

14. E. Nam, J. Kim, S. N. Guntari, H. Seyler, Q. Fu, E. H. H. Wong, A. Blencowe, D. J. Jones, F. Caruso and G. G. Qiao, Chemical Science, 2014, 5, 3374-3380.

15. B. Mizrahi, X. Khoo, H. H. Chiang, K. J. Sher, R. G. Feldman, J.-J. Lee, S. Irusta and D. S. Kohane, Langmuir, 2013, 29, 10087-10094.

16. (a) T. K. Goh, S. N. Guntari, C. J. Ochs, A. Blencowe, D. Mertz, L. A. Connal, G. K. Such, G. G. Qiao and F. Caruso, Small, 2011, 7, 28632867; (b) S. N. Guntari, T. K. Goh, A. Blencowe, E. H. H. Wong, F. Caruso and G. G. Qiao, Polymer Chemistry, 2013, 4, 68-75; (c) S. N. Guntari, E. H. H. Wong, T. K. Goh, R. Chandrawati, A. Blencowe, F. Caruso and G. G. Qiao, Biomacromolecules, 2013, 14, 2477-2483; (d) S. N. Guntari, A. C. H. Khin, E. H. H. Wong, T. K. Goh, A. Blencowe, F. Caruso and G. G. Qiao, Adv. Funct. Mater., 2013, 23, 5159-5166.

17. (a) H. Hyun and N. Yui, Macromolecular Rapid Communications, 2011, 32, 326-331; (b) J. Wu, H. He and C. Gao, Macromolecules, 2010, 43, 2252-2260.

18. (a) N. Y. Kim, N. L. Jeon, I. S. Choi, S. Takami, Y. Harada, K. R. Finnie, G. S. Girolami, R. G. Nuzzo, G. M. Whitesides and P. E. Laibinis, Macromolecules, 2000, 33, 2793-2795;

19. J. Cui, Y. Ju, K. Liang, H. Ejima, S. Lorcher, K. T. Gause, J. J. Richardson and F. Caruso, Soft Matter, 2014, 10, 2656-2663.

20. T. Ooya, M. Eguchi and N. Yui, Journal of the American Chemical Society, 2003, 125, 13016-13017. 


\section{University Library}

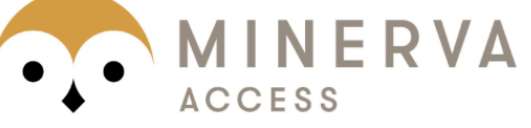

A gateway to Melbourne's research publications

Minerva Access is the Institutional Repository of The University of Melbourne

Author/s:

Tan, S;Nam, E;Cui, J;Xu, C;Fu, Q;Ren, JM;Wong, EHH;Ladewig, K;Caruso, F;Blencowe, A; Qiao, GG

Title:

Fabrication of ultra-thin polyrotaxane-based films via solid-state continuous assembly of polymers

Date:

2015-01-01

Citation:

Tan, S., Nam, E., Cui, J., Xu, C., Fu, Q., Ren, J. M., Wong, E. H. H., Ladewig, K., Caruso, F., Blencowe, A. \& Qiao, G. G. (2015). Fabrication of ultra-thin polyrotaxane-based films via solid-state continuous assembly of polymers. CHEMICAL COMMUNICATIONS, 51 (11), pp.2025-U39. https://doi.org/10.1039/c4cc08759c.

Persistent Link:

http://hdl.handle.net/11343/124160 